\title{
Safety and Efficacy of Artisan Aphakia Iris-Claw Intraocular Lens in Tertiary Hospital in Indonesia
}

\author{
Emmy Dwi Sugiarti1,2, Azalia Latuasan², Andrew M. H. Knoch ${ }^{1,2}$, Budiman Budiman ${ }^{1,2}$ \\ ${ }^{1}$ Cataract and Refractive Surgery Unit, National Eye Centre Cicendo Eye Hospital, Bandung, Indonesia \\ ${ }^{2}$ Ophtalmology Department, Faculty of Medicine Universitas Padjadjaran, Bandung, Indonesia \\ Email: emmy_ds@yahoo.com, azalia.latuasan@gmail.com
}

How to cite this paper: Sugiarti, E.D., Latuasan, A., Knoch, A.M.H. and Budiman, B. (2021) Safety and Efficacy of Artisan Aphakia Iris-Claw Intraocular Lens in Tertiary Hospital in Indonesia. Open Journal of Ophthalmology, 11, 25-35.

https://doi.org/10.4236/ojoph.2021.111003

Received: January 5, 2021

Accepted: February 6, 2021

Published: February 9, 2021

Copyright $\odot 2021$ by author(s) and Scientific Research Publishing Inc. This work is licensed under the Creative Commons Attribution International License (CC BY 4.0).

http://creativecommons.org/licenses/by/4.0/

\begin{abstract}
Introduction: Iris-claw Intraocular Lens (IOL) is one of the alternatives to correct aphakia without sufficient capsular support. This technique is preferred because it has a simple procedure. Iris-claw IOL was originally designed to be fixated on the anterior chamber. The use of retropupillary fixation is increasing because the location is more physiologic and it shows less risk to corneal endothelial damage. Purpose: To describe safety and efficacy of iris-claw Artisan IOL in correcting aphakia without sufficient capsular support. Methods: This is a descriptive retrospective study of patients with aphakic iris-claw Artisan IOL implantation in National Eye Center Cicendo Eye Hospital, Indonesia from July 2017-July 2019. Patients were divided into prepupillary and retropupillary group. The Uncorrected Visual Acuity (UCVA), Best-Corrected Visual Acuity (BCVA), Safety Index (SI), Efficacy Index (EI), and complications were recorded. The procedure is safe if SI value $\geq 1.0$ and effective if EI value $\geq 1.0$. Results: There were 54 eyes in the retropupilary group and 17 eyes in the prepupillary group. In the prepupillary group, there were $94.11 \%$ eyes with SI $\geq 1.0$, the mean SI was $1.79 \pm 1.02,50 \%$ of eyes with $\mathrm{EI} \geq 1.0$, and the mean EI was $0.77 \pm 0.20$. In the retropupillary group, there were $96.29 \%$ eyes with SI $\geq 1.0$, the mean SI was $2.49 \pm 2.23$, $74.07 \%$ of eyes with $\mathrm{EI} \geq 1.0$, and the mean EI was $1.75 \pm 1.64$. Postoperative UCVA and BCVA were improved significantly compared to preoperative visual acuity in both groups $(\mathrm{p}<0.05)$ Conclusion: Prepupillary and retropupillary iris-claw IOL implantation are safe. Retropupillary fixation technique is more effective in improving visual acuity.
\end{abstract}

\section{Keywords}

Iris-Claw, Aphakia, Safety Index, Efficacy Index 


\section{Introduction}

The ideal condition to correct aphakia is the implantation of an Intraocular Lens (IOL) in the bag [1]. Inadequate capsular support will prevent IOL implantation in the bag or sulcus and may be caused by capsular rupture, zonulysis, zonular weakness, pseudoexfoliation syndrome, pathologic myopia, ocular trauma, or Marfan syndrome [1] [2]. In such cases, alternative procedures are angle fixated, scleral fixated, iris sutured, or iris-claw IOL implantation [3] [4] [5]. Surgeon's experience, as well as eye conditions, determines the procedure of choice [3].

Implantation of angle-fixated anterior chamber IOL (AC IOL) seems easy but has some limitations and disadvantages. The main constraint is sizing. Insufficient size of IOL could damage corneal endothelium and anterior chamber angle due to rotation and/or dislocation. Excessive size of IOL gives too much pressure to the root of iris leading to damage of anterior chamber angle, the formation of peripheral anterior synechiae, an increase in intraocular pressure, and glaucoma. Angle-fixated AC IOL is also associated with chronic inflammation, corneal decompensation, cystoid macular edema, and hyphema [2] [4] [5] [6].

The scleral-fixated lens is implanted in a more physiologic location far from corneal endothelium and anterior chamber angle, but suturing technique is difficult. It requires excessive intraocular manipulation and longer surgical time. Possible complications are suture erosion, chronic inflammation, retinal detachment, vitreous and suprachoroidal hemorrhage. Suture exposure may also increase the risk of endophthalmitis [7] [8] [9].

The disadvantages of iris-sutured IOL comprise longer surgical time and the high difficulty level of technique. It also increases the risk of hypotony and intraoperative bleeding during needle passage as well as iris architecture disruption. The resulting iris damage leads to peripheral anterior synechiae, dyscoria, and limitation to dilatation [5] [10] [11] [12].

Implantation technique of iris-claw IOL is easier than iris sutured or scleral fixated lens. Haptic is fixated to midperipheral iris where the iris was less vascularized and less reactive. This location is far from corneal endothelium and iridocorneal angle, so it avoids the problem associated with IOL sizing, damage to the iris root, and anterior chamber angle. Iris-claw IOL was originally designed to be fixated on the anterior pupil (prepupillary). Retropupillary fixation later developed due to more physiologic location, far from corneal endothelium, and easier to implant than prepupillary procedure. The major disadvantage is that iris-claw IOL implantation required a $5.5 \mathrm{~mm}$ incision thus increased the risk of surgically induced astigmatism. This could be minimized using a scleral tunnel incision [4] [7] [11].

As previous studies of Indonesian aphakic iris-claw IOL have not been reported, this study aims to describe the safety and efficacy of iris-claw Artisan IOL implantations in correcting aphakic patients without adequate capsular support. 


\section{Subject and Methods}

This is a descriptive retrospective study in National Eye Center Cicendo Eye Hospital, Bandung, Indonesia. Medical records from July 2017 until July 2019 were evaluated. The study adhered to the Declaration of Helsinki and was approved by the Institutional Review Board (IRB) of Padjadjaran University. Patients' informed consent was not required by the IRB because the data were collected retrospectively. Patients' data were de-identified to protect privacy. All aphakic patients with age $>18$ years old, minimum 1 month follow-up, complete medical records, and implanted iris-claw IOL by ophthalmologist staff of Cicendo Eye Hospital are included. Exclusion criteria are an eye with a poor prognosis such as optic disc atrophy or retinal abnormalities. The intraocular lens used was aphakic iris-claw Artisan IOL (Ophtec BV, Groningen, The Netherlands) with $8.5 \mathrm{~mm}$ length, maximum tall $1.04 \mathrm{~mm}$, and optic zone-wide is $5.4 \mathrm{~mm}$. Patients were divided into retropupillary and prepupillary groups.

Indications, corneal endothelium cell count, UCVA, BCVA, safety index, efficacy index and complication were all evaluated. Primary procedure was conducted, if lens extraction and IOL implantation were done within the same time. Secondary procedure was carried out, if implantation were done at different times after lens extraction. Visual acuity was measured using the Snellen chart. Uncorrected visual acuity was examined before surgery and after surgery (1 day, 1 week, and 1 month). Best-corrected visual acuity was examined before and 1 month after surgery. Finger counting and hand movement were calculated as decimal values. Increased or decreased visual acuity was recorded if there was $\geq 2$ lines difference of the Snellen chart.

Safety index (SI) is the ratio between BCVA after and before surgery. Refractive surgery is safe if SI value $\geq 1.0$. Efficacy index (EI) is the ratio between UCVA after surgery and BCVA before surgery. Refractive surgery is effective if EI value $\geq 1.0$. [13] [14].

Objective spheris was measured using an auto refractometer RM-8900 (Topcon). Corneal endothelial cell count was measured using specular microscope SP-3000P (Topcon). A-constant used were 115 for prepupillary and 117 for retropupillary implantation. Biometry was measured using optical biometry IOL Master V.5 or ultrasound biometry TomeyBio Meter AL-100. The implantation procedure was carried out by 6 ophthalmologists while the implantation technique was chosen based on operator preference.

Statistical analysis was performed using SPSS 22.0. Data distribution in prepupillary group was tested using the Saphiro-Wilk test while in retropupillary group tested using the Kolmogorov Smirnov test. The differences of UCVA between the time of follow-up was tested using the Friedman test. The comparison between preoperative UCVA and after 1 month one was tested using the Wilcoxon test. The comparison between preoperative BCVA and after 1 month one was tested using the Wilcoxon test. Significant results were shown if $\mathrm{p}$ value $<$ 0.05 . 


\section{Results}

There were 149 eyes with aphakic iris-claw IOL implantation from Juli 2017-Juli 2019. Eligible patient eyes based on inclusion and exclusion criteria were 71 eyes from 62 patients. Patients' ages ranged from 18 to 87 years old; the mean age from the prepupillary group was $56 \pm 16.4$ years old while from the retropupillary group was $52.7 \pm 17.8$ years old. Also, there were more male subjects compared to female subjects. Most of our cases had endothelial cell count $\geq 2000$ cells $/ \mathrm{mm}^{2}$. One case from the retropupillary group had endothelial cell count of 827.5 cells $/ \mathrm{mm}^{2}$. The most common indication of primary procedure in both groups was zonular weakness $(11.76 \%$ and $14.81 \%)$. These patients had zonular weakness but examination results excluded pseudoexfoliation syndrome, Marfan syndrome, trauma, or surgical complication as underlying causes. The most common indication for the secondary procedure in both groups was an aphakic eye with inadequate capsular support after complicated cataract surgery $(52.94 \%$ and 27.77\%). Clinical characteristics are shown in Table 1.

In prepupillary group, improvement of UCVA after surgery occurred in 94.11\% $(n=16)$ eyes while it remained the same in $5.88 \%(n=1)$ eyes. Improvement of BCVA after surgery occurred in $82.35 \%(n=14)$ eyes, while it

Table 1. Clinical characteristics of the patients.

\begin{tabular}{|c|c|c|}
\hline Clinical Characteristics & $\begin{array}{l}\text { Prepupillary } \\
\text { n (\%) } \\
17 \text { eyes }\end{array}$ & $\begin{array}{c}\text { Retropupillary } \\
\text { n (\%) } \\
54 \text { eyes }\end{array}$ \\
\hline Age $($ mean \pm SD) & $56 \pm 16.4$ & $52.7 \pm 17.8$ \\
\hline \multicolumn{3}{|l|}{ Sex } \\
\hline Male & $9(52.94)$ & $48(88.89)$ \\
\hline Female & $8(47.06)$ & $6(11.11)$ \\
\hline \multicolumn{3}{|l|}{ Endothelial Cell Count (cells/mm ${ }^{2}$ ) } \\
\hline$\leq 1000$ & $0(0)$ & $1(1.85)$ \\
\hline $1000-2000$ & $5(33.33)$ & $25(46.30)$ \\
\hline$\geq 2000$ & $12(66.67)$ & $28(51.85)$ \\
\hline Primary Procedure & $4(23.52)$ & $24(44.44)$ \\
\hline Zonular weakness & $2(11.76)$ & $8(14.81)$ \\
\hline Pseudoexfoliation Syndrome & $0(0)$ & $5(9.25)$ \\
\hline Marfan syndrome & $0(0)$ & $6(11.11)$ \\
\hline Trauma & $1(5.88)$ & $3(5.55)$ \\
\hline Surgical complication & $1(5.88)$ & $2(3.70)$ \\
\hline Secondary Procedure & $13(76.48)$ & $30(55.56)$ \\
\hline Aphakia & $9(52.94)$ & $15(27.77)$ \\
\hline IOL subluxation & $3(17.65)$ & $10(18.51)$ \\
\hline IOL drop & $1(5.88)$ & $5(9.26)$ \\
\hline
\end{tabular}

IOL: Intraocular Lens. 
remained the same in $11.76 \%(\mathrm{n}=2)$ eyes, and decreased in $5.88 \%(\mathrm{n}=1)$ eye. There were $94.11 \%(\mathrm{n}=16)$ eyes with $\mathrm{SI} \geq 1.0$ and the mean SI was $1.79 \pm 1.02$. There were $50 \%(n=7)$ eyes with $\mathrm{EI} \geq 1.0$ and the mean EI was $0.77 \pm 0.20$. After surgery, $17.65 \%$ of eyes had UCVA $\geq 0.5$ and $82.35 \%$ of eyes had BCVA $\geq$ 0.5 . The comparison of UCVA before and after 1-month surgery showed a significant improvement with a p-value of 0.001 . The differences of UCVA between follow-up time can be seen in Table 2. The median UCVA increased from 0.014 to 0.32 after one month ( $\mathrm{p}$-value $<0.001$ ). The comparison of BCVA before and after 1-month surgery was significant with a p-value of 0.001 as depicted in Table 3 .

In retropupillary group, UCVA after surgery improved in $98.15 \%(\mathrm{n}=53)$ eyes and decreased in $1.85 \%(\mathrm{n}=1)$ eye. BCVA improved in $74.07 \%(\mathrm{n}=40)$ eyes, remained the same in $22.22 \%(n=12)$ eyes, and decreased in $3.70 \%(n=2)$ eyes. There were $96.29 \%(n=52)$ eyes with SI $\geq 1.0$, while the mean SI was 2.49 \pm 2.22 . There were $74.07 \%(n=40)$ eyes with EI $\geq 1.0$, while the mean EI was $1.75 \pm 1.64$. After surgery, $57.41 \%(\mathrm{n}=31)$ eyes had UCVA $\geq 0.5$ and $79.63 \%(\mathrm{n}$ $=43$ ) eyes had BCVA $\geq 0.5$. Comparisons of UCVA before and after 1-month surgery showed a significant improvement with a p-value $<0.001$. The differences of UCVA between follow-up time is shown in Table 2. The median UCVA increased from 0.014 to 0.45 after one month (p-value $<0.001$ ). The comparison of BCVA before and after 1-month surgery was significant with a p-value < 0.001 .

In our study, UCVA was significantly improved between the time of follow-up in both groups. Using SI $\geq 1.0$, retropupillary implantation was slightly safer than prepupillary $(96.29 \%$ vs $94.11 \%)$. The difference between the two groups could be seen using EI. Using EI $\geq 1.0$, retropupillary implantation gave better results than prepupillary implantation $(74.07 \%$ vs $50 \%)$.

Table 4 indicates complications in this study. The most common complication in the retropupillary group was pupil ovalization. Secondary glaucoma occurred in one eye from both groups. Corneal decompensation occurred in 1.85\% $(n=1)$ eye of the retropupillary group. One patient had haptic detachment but could be reattached successfully.

Table 2. Uncorrected visual acuity improvement between follow-up.

\begin{tabular}{|c|c|c|c|c|c|}
\hline \multirow{2}{*}{ UCVA } & \multicolumn{4}{|c|}{ Time } & \multirow{2}{*}{ p-value ${ }^{*}$} \\
\hline & Pre-operative & After 1 day & After 1 week & After 1 month & \\
\hline \multicolumn{6}{|l|}{ Prepupillary } \\
\hline Mean (SD) & $0.035(0.070)$ & $0.101(0.087)$ & $0.236(0.170)$ & $0.325(0.176)$ & \multirow{3}{*}{$<0.001$} \\
\hline Median & 0.014 & 0.08 & 0.20 & 0.32 & \\
\hline Range & $0.002-0.250$ & $0.01-0.25$ & $0.01-0.63$ & $0.05-0.70$ & \\
\hline \multicolumn{6}{|c|}{ Retropupillary } \\
\hline Mean (SD) & $0.040(0.097)$ & $0.179(0.189)$ & $0.354(0.241)$ & $0.467(0.251)$ & \multirow{3}{*}{$<0.001$} \\
\hline Median & 0.014 & 0.125 & 0.32 & 0.45 & \\
\hline Range & $0.003-0.500$ & $0.00-0.63$ & $0.01-1.00$ & $0.05-1.00$ & \\
\hline
\end{tabular}

*Friedman test; UCVA: Uncorrected Visual Acuity. 
Table 3. Best corrected visual acuity improvement after 1 month of follow-up.

\begin{tabular}{ccccccc}
\hline \multirow{2}{*}{ BCVA } & \multicolumn{3}{c}{ Prepupillary } & \multicolumn{3}{c}{ Retropupillary } \\
\cline { 2 - 6 } & $\begin{array}{c}\text { Before } \\
\text { surgery }\end{array}$ & After surgery & $\begin{array}{c}\mathrm{p} \\
\text { value }\end{array}$ & $\begin{array}{c}\text { Before } \\
\text { surgery }\end{array}$ & After surgery & $\begin{array}{c}\mathrm{p} \\
\text { value }^{*}\end{array}$ \\
\hline Mean (SD) & $0.361(0.277)$ & $0.707(0.258)$ & & $0.334(0.305)$ & $0.705(0.288)$ & \\
Median & 0.40 & 0.80 & 0.001 & 0.25 & 0.80 & $<0.001$ \\
Range & $0.005-0.800$ & $0.16-1.00$ & & $0.005-1.00$ & $0.10-1.00$ & \\
\hline
\end{tabular}

*Wilcoxon test; BCVA: Best Corrected Visual Acuity.

Table 4. Complication after 1 month of follow-up.

\begin{tabular}{ccc}
\hline Complication & $\begin{array}{c}\text { Prepupillary } \\
\mathrm{n}(\%)\end{array}$ & $\begin{array}{c}\text { Retropupillary } \\
\mathrm{n}(\%)\end{array}$ \\
\hline Pupil ovalization & $1(5.88)$ & $9(16.67)$ \\
Secondary Glaucoma & $0(0)$ & $1(1.85)$ \\
Hypotony & $0(0)$ & $1(1.85)$ \\
Uveitis & $0(0)$ & $1(1.85)$ \\
Corneal Decompensation & $0(0)$ & $1(1.85)$ \\
Detached haptic & $0(0)$ & $1(1.85)$ \\
Hyphema & $1(5.88)$ & $0(0)$ \\
\hline
\end{tabular}

\section{Discussion}

Choosing surgical techniques to correct aphakic eyes with inadequate capsular support remains challenging [2]. The technique which offers the lowest complication rate combined with the best possible visual outcome should be chosen according to patient's condition and surgeon's experience. Iris-claw Artisan IOL is an effective choice for aphakia correction with several advantages like easy to implant, minimally invasive, has shorter surgical time, and has fewer complications compared to other techniques [3] [6] [7] [11] [12].

Previous studies reported that iris-claw Artisan IOL implantation in both locations is safe to corneal endothelium but prepupillary fixation still has a small risk of endothelial touch in the shallow anterior chamber. Peralba et al. reported a greater reduction in the prepupillary group (9.6\% versus $8.7 \%)$ after 1 year [1]. Toro et al. also reported a greater reduction in the prepupillary group $(11.6 \%$ versus 9.5\%) after 5 years [15]. The difference was not significant in both studies. However, in our study retropupillary fixation in most cases is conducted to protect corneal endothelium and provide a more physiological IOL position. It is also easier to implant iris-claw Artisan IOL in retropupillary position in some cases where the procedure is combined with pars plana vitrectomy.

Some of our surgeons prefer to do prepupillary implantation with minimum corneal endothelial cell 1000 cells $/ \mathrm{mm}^{2}$. There is no consensus for minimal endothelial cell count for iris-claw IOL implantation. Toro et al. and Peralba et al. recommend doing retropupillary implantation if the endothelial cell count is less 
than 1200 cells $/ \mathrm{mm}^{2}$ [1] [15]. Toro et al. reported good results of 2 patients with endothelial cell $>900$ cells $/ \mathrm{mm}^{2}$ implanted by retropupillary iris-claw [15]. In our study, there was a patient in retropupillary group with endothelial cell count of 827.5 cells $/ \mathrm{mm}^{2}$. After surgery, BCVA in this patient was 1.0 with no corneal decompensation until the last follow-up.

Previous studies reported that aphakia iris-claw Artisan IOL implantation significantly improves visual acuity after surgery. Helvaci et al. reported that all two groups obtained a significant improvement in BCVA ( $\mathrm{p}<0.05)$ [3]. Mora et al. also reported that the groups showed comparable improvements in BCDVA after surgery (final BCVA: $0.34 \pm 0.45$ vs. $0.37 \pm 0.50 \log \mathrm{MAR}$ in the anterior and retropupillary placement groups, respectively) [16]. Toro et al. reported CDVA improved significantly in both groups ( $\mathrm{p}<0.001$, ANOVA), from the first week and during the entire follow-up ( $p<0.001$, Tukey HSD) [15]. In our study, there were also significant improvement of UCVA between follow-up time after surgery $(\mathrm{p}<0.05)$ and improvement of BCVA after 1-month follow-up ( $<<0.05)$.

Safety and efficacy are two important parameters to evaluate refractive surgery results. Based on the visual acuity of the same eye before and after surgery, we can retrospectively define the safety and effectiveness of the surgery. If postoperative BCVA is the same or more than preoperative BCVA ( $\mathrm{BI} \geq 1.0$ ), the procedure is safe. Same BCVA is considered safe because the patient did not lose their visual potential [13] [14]. In this study, patient in retropupillary group with $S I \geq$ 1.0 is slightly higher than prepupillary group (96.29\% vs $94.11 \%)$. The mean SI in the prepupillary group was $1.79 \pm 1.02$ while in the retropupillary group was $2.49 \pm 2.23$. Other studies also reported slightly better results in retropupillary group but the differences are not significant. Peralba et al. reported postoperative BCVA equal or increased in $64.7 \%$ of patients from prepupillary group and $71.4 \%$ of patients from retropupillary group [1]. Toro et al. explained that there was no difference in BCVA between the two groups after 1 month-surgery. After 5 years of follow-up, BCVA remained the same or improved in $93 \%$ of patients in the retropupillary group and $92.5 \%$ in the prepupillary group [15].

In our study, SI value in both locations was high. The mean SI in the prepupillary group was $1.79 \pm 1.02$ while in the retropupillary group was $2.49 \pm 2.23$. Ganesh et al. reported a Safety Index of 1.73 in retropupillary implantation using OV lens (Care Group, India) which may have different characteristics than Artisan IOL [17]. Correction of vision with aphakic glasses causes spherical aberrations, visual field reduction, and image size magnification. Aphakic glasses also result in disparity of image in unilateral aphakia. After IOL implantation, all of these problems were resolved, better BCVA could be achieved, resulting in a high SI value in both positions [1].

Even though both positions are safe, retropupillary implantation was more effective in our study. If the postoperative UCVA equals and even exceeds the preoperative BCVA (EI $\geq 1.0$ ), the procedure is effective [13]. This result represents an uncorrected vision after surgery at least as good as a correction with a patient's glasses or contact lens before surgery [14]. Efficacy Index $\geq 1.0$ 
occurred in $50 \%$ of eyes from the prepupillary group and in $74.07 \%$ of eyes from the retropupillary group. The mean EI in the prepupillary group was $0.77 \pm 0.2$ while in the retropupillary group was $1.83 \pm 1.66$. Eyes with low EI may still reach better BCVA after surgery. Better results in the retropupillary group might be due to more physiologic implantation location, being close to the eye's nodal point, resulting in more predictable refraction, and the procedure is less manipulative. Another possibility is in this study the number of patients is higher in the retropupillary group.

In the prepupillary group, reduced BCVA occurred in one eye. This is an aphakic eye after previous complicated cataract surgery. No retinal abnormalities in this eye but the other eye had an old retinal detachment. This eye had implanted iris-claw Artisan IOL through scleral tunnel incision near the cornea. This patient had two sutures that were too tight after surgery. Decreased BCVA was more likely due to surgical induced astigmatism. This patient only came to follow-up until 1 month. The visual acuity could be improved by releasing the sutures.

Reduction of BCVA in the retropupillary group occurred in 2 eyes. One with corneal decompensation and the other with the possibility of SIA. In an eye with corneal decompensation, endothelial cell count before surgery is $1513.6 \mathrm{cell} / \mathrm{mm}^{2}$ with hexagonality $0 \%$. Visual acuity in the fellow eye is no light perception thus we still decided to implant iris-claw IOL in retropupillary position because this eye was a last hope for vision. This patient had multiple surgeries before Artisan aphakic IOL implantation. He underwent cataract extraction as a first surgery then IOL reposition eight years later as second surgery. Five years later, he underwent IOL exchange to AC IOL as third surgery. By the time, the AC IOL decentered again. Instability of decentered IOL with endothelial touch, improper AC-IOL, and multiple surgical procedures and manipulation could reduce the number of endothelial cells, lead to persistent corneal edema [18] [19] [20].

In an eye with the possibility of SIA, previous surgery done was complicated phacoemulsification converted to extracapsular cataract extraction using temporal corneal incision. This eye was left in aphakic condition and had 4 sutures after surgery. After two months, iris-claw Artisan IOL was implanted in this eye with the addition of three sutures. In this eye, BCVA after 1-month surgery decreased to 4 lines of the Snellen chart. Corneal incision induced greater SIA compared with scleral tunnel incision. Martinez et al. reported Surgically Induced Astigmatism (SIA) in the scleral tunnel incision group (0.73 \pm 0.62 diopter) was significantly lower than in the corneal incision group $(2.49 \pm 1.36$ diopter) $(\mathrm{p}<$ $0.001)$ [11] [12] [21].

The most common complication in this study is pupil ovalization. It did not influence postoperative BCVA. Ovalization may occur because of the tightness or asymmetric enclavation in midperipheral iris stroma. This condition tends to normalize over time, usually within 1 week but sometimes longer [7] [15] [22]. In this study, pupil ovalization occurred in $5.88 \%(\mathrm{n}=1)$ eyes from the prepupillary group and $16.67 \%(n=9)$ eyes from the retropupillary group after 1 
month follow-up. Martinez et al. reported pupil ovalization occurred in $11.8 \%$ of eyes but reversed within 6 month follow-up [11]. Gonnermann et al. reported pupil ovalization in $24.8 \%$ of eyes within first week after retropupillary implantation but persistent in only $13.9 \%$ eyes [5]. Persistent ovalization could be caused by misplaced IOL centration during enclavation resulting in the claw does not pinch midperipher iris but instead pinches the sphincter muscle of the iris. This study only follows up patients until 1-month post-surgery. Further study with a longer follow-up time is needed.

Secondary glaucoma occurred in $1.85 \%(\mathrm{n}=1)$ of eye from the retropupillary group after 1-month follow-up with a history of Pseudoexfoliation syndrome. Increased IOP is due to preexisting condition. Guell et al. reported significant IOP changes only in patients with pigment dispersion or pseudoexfoliation [23]. Toro et al. reported increased IOP in one patient from each group after 3 years of follow-up but they had a family history of primary open-angle glaucoma [15]. In our study, high IOP could be controlled by medication. Hypotony occurred in one eye with history of pars plana vitrectomy. The IOP was normalized after one week follow up without any intervention. Anterior uveitis occurred in one eye but the inflammation could be controlled by medication. Haptic detachment occurred in one eye but after reposition of the detached haptic the result is good.

\section{Conclusion}

In conclusion, iris-claw IOL implantation in both positions is safe but retropupillary implantation is more effective in increasing visual acuity. This is a preliminary study that used previously recorded data. This study has several limitations such as this is a retrospective study thus we only have limited complete medical records. Most of our patients only follow up for 1 month after surgery. In this study, implantation was performed by six surgeons. There are some variations in implantation technique and location preference between different surgeons. The subgroup sample is also small and unevenly distributed between prepupillary and retropupillary groups, so the results between the two groups are not statistically comparable. Further prospective studies with more homogenous samples and longer follow-up time could be done to give more accurate results.

\section{Conflicts of Interest}

The authors declare no conflicts of interest regarding the publication of this paper.

\section{References}

[1] Touriño Peralba, R., Lamas-Francis, D., Sarandeses-Diez, T., Martínez-Pérez, L. and Rodríguez-Ares, T. (2018) Iris-Claw Intraocular Lens for Aphakia: Can Location Influence the Final Outcomes? Journal of Cataract \& Refractive Surgery, 44, 818-826. https://doi.org/10.1016/j.jcrs.2018.05.010

[2] Schallenberg, M., Dekowski, D., Hahn, A., Laube, T., Steuhl, K.-P. and Meller, D. 
(2014) Aphakia Correction with Retropupillary Fixated Iris-Claw Lens (Artisan): Long-Term Results. Clinical Ophthalmology, 8, 137-141. https://doi.org/10.2147/OPTH.S55205

[3] Helvaci, S., Demirduzen, S. and Oksuz, H. (2016) Iris-Claw Intraocular Lens Implantation: Anterior Chamber versus Retropupillary Implantation. Indian Journal of Ophthalmology, 64, 45-49. https://doi.org/10.4103/0301-4738.178139

[4] Güell, J.L., Verdaguer, P., Mateu-Figueras, G., Elies, D., Gris, O., Amich, J.M., et al. (2016) Unilateral Iris-Claw Intraocular Lens Implantation for Aphakia: A Paired-Eye Comparison. Cornea, 35, 1326-1332. https://doi.org/10.1097/ICO.0000000000001000

[5] Gonnermann, J., Klamann, M.K.J., Maier, A.-K., Rjasanow, J., Joussen, A.M., Bertelmann, E., et al. (2012) Visual Outcome and Complications after Posterior Iris-Claw Aphakic Intraocular Lens Implantation. Journal of Cataract \& Refractive Surgery, 38, 2139-2143. https://doi.org/10.1016/j.jcrs.2012.07.035

[6] Brunin, G., Sajjad, A., Kim, E.J., Montes de Oca, I., Weikert, M.P., Wang, L., et al. (2017) Secondary Intraocular Lens Implantation: Complication Rates, Visual Acuity, and Refractive Outcomes. Journal of Cataract \& Refractive Surgery, 43, 369-376. https://doi.org/10.1016/j.jcrs.2016.12.024

[7] Choragiewicz, T., Rejdak, R., Grzybowski, A., Nowomiejska, K., Moneta-Wielgoś, J., Ozimek, M., et al. (2016) Outcomes of Sutureless Iris-Claw Lens Implantation. Journal of Ophthalmology, 2016, Article ID: 7013709.

https://doi.org/10.1155/2016/7013709

[8] Baykara, M., Ozcetin, H., Yilmaz, S. and Timuçin, Ö.B. (2007) Posterior Iris Fixation of the Iris-Claw Intraocular Lens Implantation through a Scleral Tunnel Incision. American Journal of Ophthalmology, 144, 586-591.E2. https://doi.org/10.1016/j.ajo.2007.06.009

[9] Madhivanan, N., Sengupta, S., Sindal, M., Nivean, P.D., Kumar, M.A. and Ariga, M. (2019) Comparative Analysis of Retropupillary Iris Claw versus Scleral-Fixated Intraocular Lens in the Management of Post-Cataract Aphakia. Indian Journal of Ophthalmology, 67, 59-63. https://doi.org/10.4103/ijo.IJO $326 \quad 18$

[10] Holt, D.G., Young, J., Stagg, B. and Ambati, B.K. (2012) Anterior Chamber Intraocular Lens, Sutured Posterior Chamber Intraocular Lens, or Glued Intraocular Lens: Where Do We Stand? Current Opinion in Ophthalmology, 23, 62-67. https://doi.org/10.1097/ICU.0b013e32834cd5e5

[11] Hernández Martínez, A. and Almeida González, C.V. (2018) Iris-Claw Intraocular Lens Implantation: Efficiency and Safety According to Technique. Journal of Cataract \& Refractive Surgery, 44, 1186-1191. https://doi.org/10.1016/j.jcrs.2018.06.049

[12] Khan, M.J., Al-Mohtaseb, Z.N. and Kim, D.B. (2018) Secondary IOLs: ACIOL vs Iris Sutured vs Scleral Fixated vs Phakic IOL in Aphakic Settings. Current Ophthalmology Reports, 6, 217-225. https://doi.org/10.1007/s40135-018-0184-1

[13] Linke, S.J. and Katz, T. (2016) Complications in Corneal Laser Surgery. Springer, Cham, 12-13. https://doi.org/10.1007/978-3-319-41496-6

[14] Kertes, P.J. and Johnson, M.T. (2014) Evidence-Based Eye Care. 2nd Edition, Lippincott Williams and Wilkins, Philadelphia, $79 \mathrm{p}$.

[15] Toro, M.D., Longo, A., Avitabile, T., Nowomiejska, K., Gagliano, C., Tripodi, S., et al. (2019) Five-Year Follow-Up of Secondary Iris-Claw Intraocular LENS implantation for the Treatment of Aphakia: Anterior Chamber versus Retropupillary Implantation. PLoS ONE, 14, e0214140. https://doi.org/10.1371/journal.pone.0214140 
[16] Mora, P., Calzetti, G., Favilla, S., Forlini, M., Tedesco, S., Date, P., et al. (2018) Comparative Analysis of the Safety and Functional Outcomes of Anterior versus Retropupillary Iris-Claw IOL Fixation. Journal of Ophthalmology, 2018, Article ID: 8463569. https://doi.org/10.1155/2018/8463569

[17] Ganesh, S., Brar, S. and Relekar, K. (2016) Long Term Clinical and Visual Outcomes of Retrofixated Iris Claw Lenses Implantation in Complicated Cases. Open Access Journal of Clinical Ophthalmology, 10, 111-118.

https://doi.org/10.2174/1874364101610010111

[18] Sharma, N., Singhal, D., Nair, S.P., Sahay, P., Sreeshankar, S. and Maharana, P.K. (2017) Corneal Edema after Phacoemulsification. Indian Journal of Ophthalmology, 65, 1381-1389. https://doi.org/10.4103/ijo.IJO $871 \quad 17$

[19] Yi, D.H. and Dana, M.R. (2002) Corneal Edema after Cataract Surgery: Incidence and Etiology. Seminars in Ophthalmology, 17, 110-114.

https://doi.org/10.1076/soph.17.3.110.14783

[20] Pricopie, S., Istrate, S., Voinea, L., Leasu, C., Paun, V. and Radu, C. (2017) Pseudophakic Bullous Keratopathy. Romanian Journal of Ophthalmology, 61, 90-94. https://doi.org/10.22336/rjo.2017.17

[21] Kwon, H.J., Nam, S.M., Stulting, R.D., Lim, C.Y. and Seo, K.Y. (2014) Comparison of Surgically Induced Astigmatism Following Iris-Claw PIOL Insertion with Scleral, Limbal, or Corneal Incisions. Journal of Refractive Surgery, 30, 330-335. https://doi.org/10.3928/1081597X-20140416-02

[22] Forlini, M., Soliman, W., Bratu, A., Rossini, P., Cavallini, G.M. and Forlini, C. (2015) Long-Term Follow-Up of Retropupillary Iris-Claw Intraocular Lens Implantation: A Retrospective Analysis. BMC Ophthalmology, 15, Article No. 143. https://doi.org/10.1186/s12886-015-0146-4

[23] Güell, J.L., Verdaguer, P., Elies, D., Gris, O., Manero, F., Mateu-Figueras, G., et al. (2014) Secondary Iris-Claw Anterior Chamber Lens Implantation in Patients with Aphakia without Capsular Support. British Journal of Ophthalmology, 98, 658-663. https://doi.org/10.1136/bjophthalmol-2013-304035 